Case Report

\title{
Estimating the Radiation Dose to the Fetus in Prophylactic Internal Iliac Artery Balloon Occlusion: Three Cases
}

\author{
Kentaro Kai, ${ }^{1}$ Tomohiro Hamada, ${ }^{2}$ Akitoshi Yuge, ${ }^{1}$ Hiro Kiyosue, ${ }^{2}$ Yoshihiro Nishida, ${ }^{1}$ \\ Kaei Nasu, ${ }^{1}$ and Hisashi Narahara ${ }^{1}$ \\ ${ }^{1}$ Department of Obstetrics and Gynecology, Oita University Faculty of Medicine, Oita 879-5593, Japan \\ ${ }^{2}$ Department of Radiology, Oita University Faculty of Medicine, Oita 879-5593, Japan \\ Correspondence should be addressed to Kentaro Kai; kenta9sp@oita-u.ac.jp
}

Received 6 February 2015; Revised 27 May 2015; Accepted 28 May 2015

Academic Editor: Loïc Sentilhes

Copyright (C) 2015 Kentaro Kai et al. This is an open access article distributed under the Creative Commons Attribution License, which permits unrestricted use, distribution, and reproduction in any medium, provided the original work is properly cited.

\begin{abstract}
Background. Although radiation exposure is of great concern to expecting patients, little information is available on the fetal radiation dose associated with prophylactic internal iliac artery balloon occlusion (IIABO). Here we estimated the fetal radiation dose associated with prophylactic IIABO in Caesarean section (CS). Cases. We report our experience with the IIABO procedure in three consecutive patients with suspected placenta previa/accreta. Fetal radiation dose measurements were conducted prior to each CS by using an anthropomorphic phantom. Based on the simulated value, we calculated the fetal radiation dose as the absorbed dose. We found that the fetal radiation doses ranged from 12.88 to $31.6 \mathrm{mGy}$. The fetal radiation dose during the prophylactic IIABOs did not exceed $50 \mathrm{mGy}$. Conclusion. The IIABO procedure could result in a very small increase in the risk of harmful effects to the fetus.
\end{abstract}

\section{Introduction}

Over the past decade, the procedure known as prophylactic internal iliac artery balloon occlusion (IIABO) for suspected placenta previa/accreta has become more frequently performed against a background of significant associated morbidity, uncertainties in antenatal diagnosis, the desire for fertility preservation, and advanced interventional radiologic techniques [1].

However, little information is available on the fetal radiation dose associated with prophylactic IIABO. The IIABO procedure is performed under fluoroscopic guidance. There are potential risks related to ionizing radiation, and fetal radiation exposure is of great concern to expecting patients because fetal tissues are more susceptible to radiation injury [2]. In view of the possibility of radiation-induced cancer in the fetus [3], a fetal dose estimate and a critical risk-benefit analysis for the mother and the fetus must be conducted prior to the initiation of the IIABO procedure [4].

The purposes of the present study were to (1) estimate the fetal radiation dose associated with prophylactic IIABO during Caesarean section (CS) and (2) report our experience with this procedure in three patients with suspected placenta previa/accreta.

\section{Case Presentation}

Three patients for whom sonographic and MRI findings revealed possible placenta previa/accreta were scheduled to undergo CS deliveries. Because of the high probability of major hemorrhage and the potential need for a cesarean hysterectomy in all three patients, each of the patients was referred to our interventional radiology department for the temporary placement of occlusive balloon catheters in the internal iliac arteries before her CS delivery and possible hysterectomy. We conducted a geometrical estimation to obtain phantom dose measurements as close as possible to the actual values, using the same equipment as that used for the patients, and then we used the simulated values to estimate the radiation dose to the fetus.

2.1. Fetal Radiation Dose Simulation. We simulated two scenarios: (1) the patient was catheterized in the angiography 
TABLE 1: Simulated results of radiation exposure with the Infinix Active DSA system in the angiography suite.

\begin{tabular}{|c|c|c|c|c|c|}
\hline \multirow{2}{*}{ I.I. Size } & \multirow{2}{*}{ Mode } & \multicolumn{2}{|c|}{ Fetus } & \multicolumn{2}{|c|}{ Skin } \\
\hline & & Dose rate $(\mathrm{mGy} / \mathrm{min})$ & $\mathrm{DSA}^{*}(\mathrm{mG} \mathrm{y} / \mathrm{f})$ & Dose rate (mGy/min) & $\mathrm{DSA}^{*}(\mathrm{mGy} / \mathrm{f})$ \\
\hline \multirow{5}{*}{14 inches } & Normal & 7.0 & NA & 54.4 & NA \\
\hline & Middle & & & & \\
\hline & $15 \mathrm{f} / \mathrm{s}$ & 4.6 & NA & 38.2 & NA \\
\hline & $12 \mathrm{f} / \mathrm{s}$ & 3.6 & 0.5 & 30.2 & NA \\
\hline & Low & 2.0 & NA & 17.4 & NA \\
\hline
\end{tabular}

* The pulsed fluoroscopy rate was 24 fps with $12 \mathrm{sec} \mathrm{image} \mathrm{acquisition.} \mathrm{I.I.,} \mathrm{image} \mathrm{intensifier;} \mathrm{f/s,} \mathrm{frame} \mathrm{per} \mathrm{second;} \mathrm{DSA,} \mathrm{digital} \mathrm{subtraction} \mathrm{angiography;} \mathrm{NA,}$ not available.

TABLE 2: Simulated results of radiation exposure with the Siemens Siremobil Compact C-arm in the operating suite.

\begin{tabular}{|c|c|c|c|c|c|}
\hline \multirow{2}{*}{ I.I. Size } & \multirow{2}{*}{ Mode } & \multicolumn{2}{|c|}{ Fetus } & \multicolumn{2}{|c|}{ Skin } \\
\hline & & Dose rate $(\mathrm{mGy} / \mathrm{min})$ & $\mathrm{DSA}^{*}(\mathrm{mGy} / \mathrm{f})$ & Dose rate $(\mathrm{mGy} / \mathrm{min})$ & $\mathrm{DSA}^{*}(\mathrm{mG} / \mathrm{f})$ \\
\hline 9 inches & Normal & 3.4 & NA & 46 & NA \\
\hline
\end{tabular}

${ }^{*}$ Previously performed in angiography suite, DSA in the operating suite was unplanned in this simulation. Abbreviations are as in Table 1.

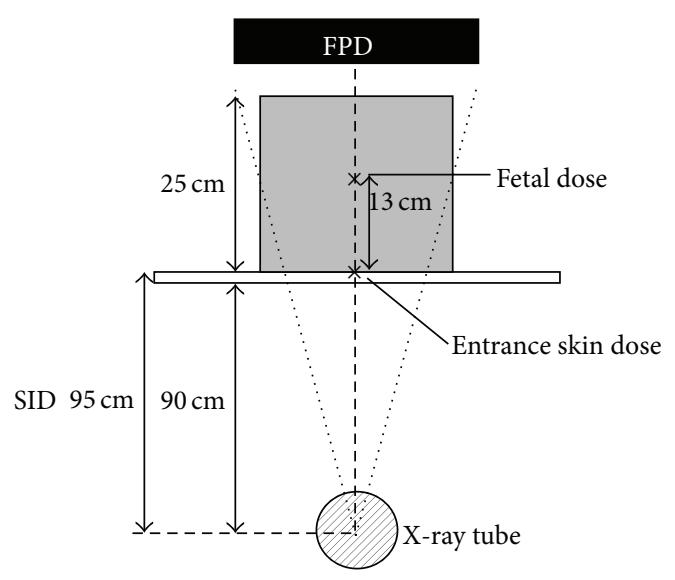

FIGURE 1: Schematic of the acrylic phantom used to simulate the fetal radiation dose in the angiography suite. FDP, flat-panel detector; SID, source-image distance.

suite and then transferred to an operating suite and (2) the patient was catheterized in the operating suite. Fetal radiation dose measurements were obtained using an anthropomorphic phantom made by a $10 \mathrm{~mm}$ thick acrylic board (41436000 XAC-type 1, Kyoto Kagaku, Kyoto, Japan). Figure 1 is a schematic diagram of the acrylic phantom used to simulate the fetal radiation dose in the angiography suite. To simulate pregnancy in the third trimester, we adjusted the overall thickness of the phantom to $25 \mathrm{~cm}$. Measurements were made on an Infinix Active digital subtraction angiography (DSA) system (Toshiba, Tokyo). We measured the entrance skin dose as the interventional reference point (i.e., the isocenter). We measured the fetal dose using a manufacturer-calibrated skin dose monitor (SDM 104-101 V4.0, McMahon Medical, San Diego, CA) at $13 \mathrm{~cm}$ from the isocenter; the fetus was assumed to lie at about the center of the phantom [5]. The $5 \mathrm{~cm}$ thick board was located $90 \mathrm{~cm}$ from the X-ray tube, and the source-image distance was $95 \mathrm{~cm}$. The simulation results are shown in Table 1. The table's column heading

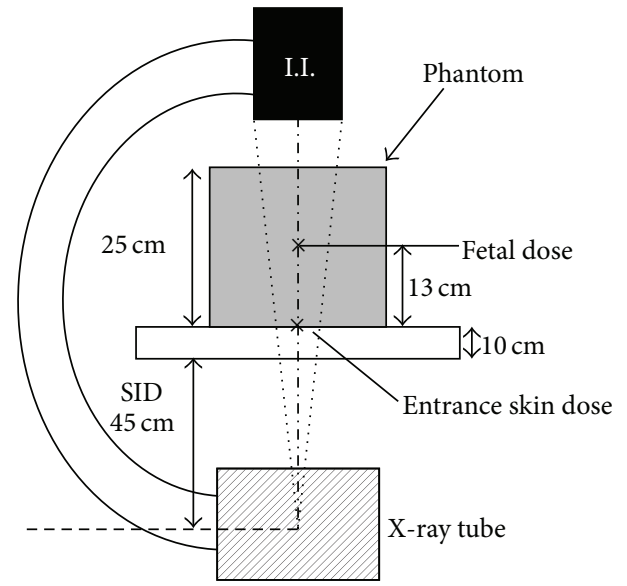

FIgURE 2: The X-ray tube-image intensifier C-arm in relation to the phantom used to simulate the fetal radiation dose in the operating suite. I.I., image intensifier.

for the fluoroscopy "Mode" was as follows. The "Normal" mode provided high X-ray output and high image quality, and the "Low" mode provided low X-ray output and low image quality. These modes were selectivity used based on the operator's need.

Figure 2 is a schematic of the X-ray tube image intensifier $\mathrm{C}$-arm in relation to the phantom in the operating suite. The irradiation was carried out using a Siremobil Compact L Carm (Siemens, Erlangen, Germany) or a GE-OEC 9800 Carm (GE Healthcare, Buckinghamshire, UK) with last-image hold ability. The height of the operating table was leveled with the usual lithotomy position. The X-ray tube voltage and current used with the Siremobil Compact L C-arm were $106 \mathrm{kV}$ and $3.1 \mathrm{~mA}$, respectively. With the GE-OEC $9800 \mathrm{C}-$ arm, the X-ray tube voltage and current were automatically regulated. The simulation results are shown in Tables 2 and 3.

The Institutional Review Board of the Oita University Faculty of Medicine approved this single-center retrospective 
TABLE 3: Simulated results of radiation exposure with the GE-OEC C-arm in the operating suite.

\begin{tabular}{|c|c|c|c|c|c|}
\hline \multirow{2}{*}{ I.I. Size } & \multirow{2}{*}{ Mode } & \multicolumn{2}{|c|}{ Fetus } & \multicolumn{2}{|c|}{ Skin } \\
\hline & & Dose rate (mGy/min) & $\mathrm{DSA}^{*}(\mathrm{mGy} / \mathrm{f})$ & Dose rate $(\mathrm{mGy} / \mathrm{min})$ & $\mathrm{DSA}^{*}(\mathrm{mGy} / \mathrm{f})$ \\
\hline \multirow{2}{*}{12 inches } & Normal & 5.6 & 2.4 & 42.2 & 22.0 \\
\hline & Low & 2.0 & 1.4 & 16.6 & 11.6 \\
\hline
\end{tabular}

${ }^{*}$ The pulsed fluoroscopy rate was $8 \mathrm{fps}$ with $10 \mathrm{sec}$ image acquisition. Abbreviations are as in Table 1.

study, and all three patients provided written informed consent.

2.2. Endovascular Technique. Arterial access to both femoral arteries was gained using the percutaneous Seldinger technique under local regional anesthesia with lidocaine. Two occlusion balloon catheters (5-Fr Moiyan; Miyano Medical Instruments, Kobe, Japan) were placed in the most proximal division of the internal iliac arteries through 5-Fr crossover sheaths (Terumo, Tokyo) in the contralateral femoral artery. Sufficient diluted contrast medium (usually approximately $1.0 \mathrm{~mL}$ ) to occlude the arteries was registered. Fluid flow stagnation with an inflated balloon (dia. $11 \mathrm{~mm}$ ) was confirmed. Sheaths and balloon catheters were fixed with skin sutures and a drape cover.

Following the cramping of the umbilical cord, the occlusion balloons were blindly inflated. During the entire surgical procedure, the femoral introducer sheaths were flushed with heparinized solution (5,000 IU heparin in $500 \mathrm{~mL}$ of saline) using an intermittent bolus technique at $10 \mathrm{~min}$ intervals. The balloons were inflated intermittently, after which the CS was performed to confirm adequate hemostasis. The sheaths and balloon catheters were removed immediately after the surgery.

2.3. Patients. Table 4 provides information about the patient's surgeries and outcomes. The patients' cases are briefly summarized here.

Case 1. A 25-year-old woman was referred to our hospital at $31 \mathrm{wks}$ of pregnancy for suspected placenta previa. She had had two uterine curettages for spontaneous abortion. At 36 wks she underwent a bilateral ureteral stent placement for the prevention of intraoperative ureteral injury during a possible emergency Caesarean hysterectomy. On the morning of the elective CS, bilateral catheterization of the internal iliac arteries was performed in an angiography suite, using the Infinix Active DSA system. After the patient's transfer to the operating suite (scenario \#1), we confirmed the position of the balloons using Siremobil Compact L C-arm fluoroscopy. After the cesarean delivery, the sleeping infant needed life support by neonatologists in the neonatal intensive care unit (NICU). The placenta was separated. The final histological examination showed no findings of placenta accreta. The mother and infant left the hospital 10 days after the CS, without complications.

The fluoroscopy with the Infinix Active DSA system in the angiography suite was performed using the condition of middle mode- $12 \mathrm{f} / \mathrm{s}$. The subtotal radiation dose in the angiography suite was $19.7 \mathrm{mGy}$. After the patient's transfer to the operating suite, the position of the balloons needed to be confirmed by Siemens Siremobil Compact C-arm fluoroscopy. The total fetal radiation dose was $31.6 \mathrm{mGy}$ (Table 5). We followed the infant to 4 yrs of age. We detected no congenital defects that could be related to the radiation dose.

Case 2. A 37-year-old woman was evaluated at $28 \mathrm{wks}$ for bleeding and uterine contractions. Nine months earlier, she had undergone debulking surgery-reducing the massfor adenomyosis, adhesiolysis for pelvic endometriosis, and right uterosacral nerve ablation for chronic pelvic pain. She became pregnant during the third trial of in vitro fertilization and embryo transfer (IVF-ET). At $32 \mathrm{wks}$, considering the recurrence of bleeding and the significant drop in the patient's hemoglobin (from 11.7 to $8.8 \mathrm{gm} / \mathrm{dL}$ ), an emergency CS was scheduled. Bilateral ureteral stents were placed preoperatively. Immediately after a bilateral catheterization of the internal iliac arteries, the emergency CS was performed with rapid sequence induction. We performed all angiographic and surgical procedures in the operating suite using the GEOEC C-arm (scenario \#2). The placenta separated, but a $3 \times$ $3-\mathrm{cm}$ fragment of the placenta was firmly adherent to the posterior lower segment of the uterus.

Following medical hemostasis with a local injection of diluted Dinoprost, the balloons were deflated. Active hemorrhaging from the retained placenta resumed, and thus the balloons were inflated and four points of surgical hemostasis with $\# 0$ absorbable sutures were performed. After hemostasis, the retained placenta was left in situ. The sleeping infant needed $7 \mathrm{wks}$ of neonatal intensive care. The mother left the hospital 3 wks after the CS, without complications. The delivered placenta showed no findings of placenta accreta histologically.

The fluoroscopy with the GE-OEC C-arm was performed using the normal mode. The total fetal radiation dose was $18.0 \mathrm{mGy}$ (Table 5). The infant had isolated congenital patent ductus arteriosus, and the initial pharmacologic therapy succeeded in closing it. We followed the infant to $5 \mathrm{yrs}$ of age, and she remained healthy.

Case 3. A 42-year-old woman was transferred to our hospital at 26 wks for uncontrolled bleeding after cervical polypectomy. She had had two uterine curettages for one miscarriage and one first-trimester abortion. She became pregnant during the first trial of IVF-ET after a debulking surgery for adenomyosis. At 37 wks an elective CS was performed with 


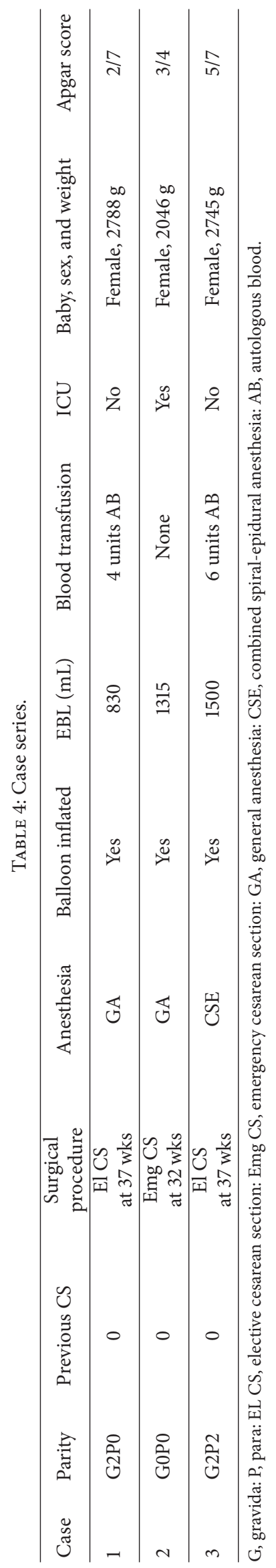


TABLE 5: Estimated fetal radiation dose in our patients.

\begin{tabular}{|c|c|c|c|c|}
\hline Patient number & $\begin{array}{c}\text { Fluoroscopy } \\
(\mathrm{mGy} / \mathrm{min} \times \mathrm{min})\end{array}$ & DSA (mGy/f × times) & Subtotal (mGy) & Total (mGy) \\
\hline \multirow{2}{*}{1} & $3.6 \times 5.2=18.7^{*}$ & \multirow{2}{*}{$0.5 \times 2=1.0^{*}$} & 19.7 & \\
\hline & $3.4 \times 3.5=11.9^{\dagger}$ & & 11.9 & 31.6 \\
\hline 2 & $2.0 \times 5.4=10.8$ & $2.4 \times 3=7.2$ & 18.0 & 18.0 \\
\hline 3 & $2.0 \times 5.32=10.64$ & $1.4 \times 4=2.24$ & 12.88 & 12.88 \\
\hline
\end{tabular}

${ }^{*}$ Angiography suite. ${ }^{\dagger}$ Operating suite.

TABLE 6: Summary of studies of the fetal radiation dose from prophylactic balloon catheterization.

\begin{tabular}{lcccc}
\hline Reference & Year & Patients $(n)$ & Occlusion site & Fetal radiation dose (mGy) \\
\hline $\begin{array}{l}\text { Levine et al. [6] } \\
\text { Bodner et al. [7] }\end{array}$ & 1999 & 9 & $\begin{array}{c}\text { Internal iliac }(n=7) \\
\text { and uterine }(n=2) \\
\text { arteries }\end{array}$ & 61 (median of 9 cases) \\
\hline Yi et al. [8] & 2006 & 3 & Internal iliac arteries & 32 (mean of 3 cases) \\
\hline This study & 2010 & 1 & Internal iliac arteries & 55 \\
\hline
\end{tabular}

combined epidural and general anesthesia without ureteral stent placement. We performed all angiographic and surgical procedures in the operating suite using the GE-OEC C-arm (scenario \#2). The infant needed 2 wks of life support in the NICU. The placenta separated. The final histological examination showed no findings of placenta accreta. The mother left the hospital 2 wks after the CS, without complications.

The fluoroscopy with the GE-OEC C-arm was performed in the low mode. The total fetal radiation dose was $12.88 \mathrm{mGy}$ (Table 5). We followed the infant to $5 \mathrm{yrs}$ of age, and she remained healthy.

\section{Discussion}

Our study produced two observations. First, based on the simulated and practical data, we obtained the fetal radiation doses during prophylactic IIABO. The fetal radiation dose during these three prophylactic IIABO procedures ranged from 12.88 to $31.6 \mathrm{mGy}$. The average fetal radiation dose in our patients was $21 \mathrm{mGy}$. Second, we observed that performing the angiographic and surgical procedures at the same site reduced the fetal exposure to radiation.

Among the articles that we reviewed, only three studies [6-8] reported the fetal radiation dose associated with prophylactic IIABO (Table 6). Compared to these reports, our study showed minimal fetal radiation exposure. These results raise the possibility that the angiographic procedures used in the prior studies had an effect on the fetal radiation exposure, since in the prior studies all of the angiographic procedures were performed in the angiographic suite, and then the patients were transferred to an operating suite for the CS.

In their practice guidelines, The American College of Obstetricians and Gynecologists [3] notes that the risks of fetal effects, including childhood cancer induction, are thought to be small at a dose of $100 \mathrm{mGy}$ and to be negligible at doses $<50 \mathrm{mGy}$. The U.S. National Council on Radiation Protection (NCRP) stated that, even for a highly unlikely $50 \mathrm{mSv}$ dose-the X-ray radiation weighting factor of which is 1.0 - to a pregnant woman, the risk is small compared to other risks to the fetus [9]. The International Commission on Radiological Protection (ICRP) estimates a $0.06 \%$ increased absolute cumulative risk of fetal cancer for each additional $10 \mathrm{mGy}$ after a child aged 0 to 15 years old receives wholebody radiation [10]. Based on the ICRP report, the absolute cumulative risk of cancer among our patients ranged from 0.8 to $1.9 \%$.

Neel et al. [11] reported that in cohorts totaling over six million children across a 40 -year period, that is, the children of survivors of the atomic bombings in Hiroshima and Nagasaki and the children of a suitable control population, no statistically significant effects of radiation emerged with respect to malignancies in the subjects up to 20 years old. Although the long-term effects of radiation are not known, given the current evidence, the risk to the fetus from the doses obtained by our simulation appears negligible.

Performing the angiographic and surgical procedures in the same suite reduced the fetal exposure to radiation. Limiting the fluoroscopy time is one of the most important steps to reduce the radiation dose [12]. Case 1, the first case in our institutional experience, was catheterized under fluoroscopic guidance in the angiography suite and then transferred to the operating suite for her CS. Although catheterization in the angiography suite has many benefits with respect to the X-ray output, image quality, and other types of support equipment for interventional radiology, we had to reconfirm the position of the IIABO catheters in the operating suite. The management of Case 1 initiated a change of practice in our unit.

In Cases 2 and 3, all CS procedures were performed in the operating suite, with the interventional radiologist placing 


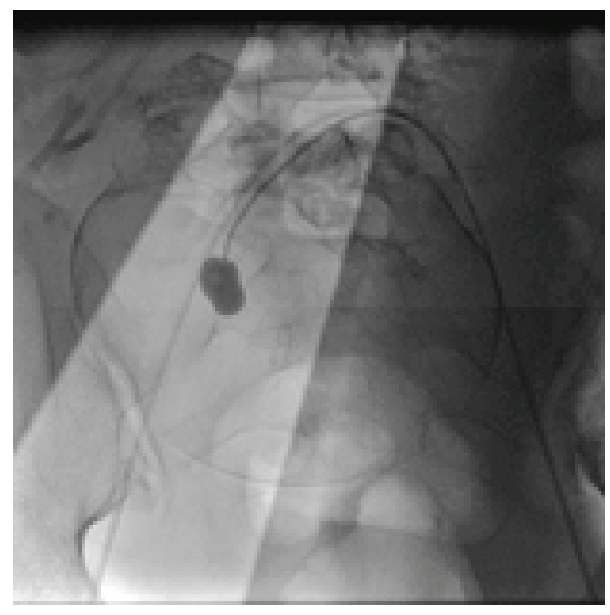

(a)

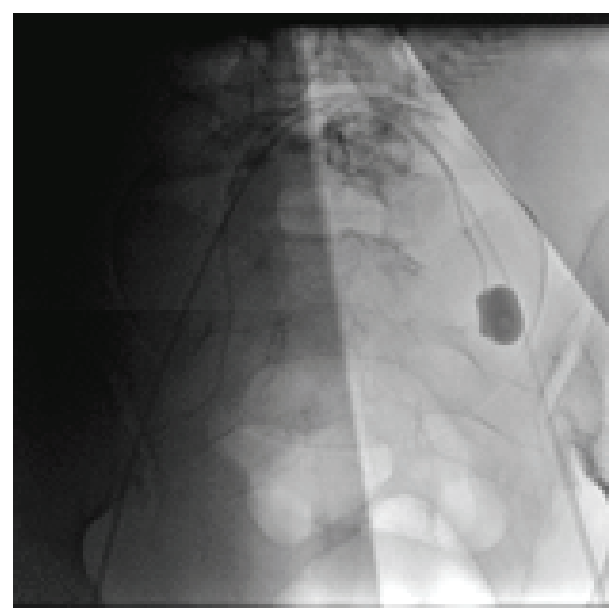

(b)

FIGURE 3: A series of images acquired during the placement of the catheters using shielding. (a) right internal iliac artery and (b) left internal iliac artery.

the IIABO catheters using the C-arm, resulting in a lower total radiation dose. In therapeutic radiology, steps should also be taken to minimize the radiation beam so that it affects only the area of interest [13]. We used shielding to ensure minimal fetal radiation exposure during the placement of the internal iliac catheters (Figures 3(a) and 3(b)).

Some limitations of our study warrant consideration. First, we overestimated the risk of placenta accreta in our cohort. There are still uncertainties in the antenatal diagnosis of placenta accreta [14], but preparations for massive peripartum blood loss and extended surgery are an important precaution. Second, the different radiological technique and the presence or absence of adherent placenta had some effect on the fetal radiation dose. The technical differences between first case and the others was the additional fluoroscopy to confirm the position of the balloons, and all fluoroscopic procedures were completed preoperatively. The estimated fetal radiation dose obtained in our case series was a relatively validated measurement.
In conclusion, we identified two important clinical issues. The estimated fetal radiation dose during prophylactic IIABO did not exceed $50 \mathrm{mGy}$. Performing angiographic and surgical procedures in the operating suite under C-arm fluoroscopy can avoid the need for a reconfirmation of the balloon catheters' position and is a potential approach to reduce fetal exposure to radiation. Although further reports should be accumulated to determine the long-term prognosis of the fetus, the IIABO procedure could result in a very small increase in the risk of harmful effects to the fetus.

\section{Conflict of Interests}

The authors declare that there is no conflict of interests regarding the publication of this paper.

\section{References}

[1] J. Sadashivaiah, R. Wilson, A. Thein, H. McLure, C. J. Hammond, and G. Lyons, "Role of prophylactic uterine artery balloon catheters in the management of women with suspected placenta accreta," International Journal of Obstetric Anesthesia, vol. 20, no. 4, pp. 282-287, 2011.

[2] N. Campbell, K. Sparrow, M. Fortier, and T. Ponich, "Practical radiation safety and protection for the endoscopist during ERCP," Gastrointestinal Endoscopy, vol. 55, no. 4, pp. 552-557, 2002.

[3] ACOG Committee on Obstetric Practice, "ACOG Committee Opinion. Number 299, September 2004 (replaces No. 158, September 1995). Guidelines for diagnostic imaging during pregnancy," Obstetrics \& Gynecology, vol. 104, no. 3, pp. 647-651, 2004.

[4] American College of Radiology, ACR Practice Guideline for Imaging Pregnant or Potentially Pregnant Adolescents and Women with Ionizing Radiation, American College of Radiology, Reston, Va, USA, 2008.

[5] V. Ioffe, R. S. Hudes, D. Shepard, J. M. Simard, L. S. Chin, and C. Yu, "Fetal and ovarian radiation dose in patients undergoing gamma knife radiosurgery," Surgical Neurology, vol. 58, no. 1, pp. 32-41, 2002.

[6] A. B. Levine, K. Kuhlman, and J. Bonn, "Placenta accreta: comparison of cases managed with and without pelvic artery balloon catheters," Journal of Maternal-Fetal and Neonatal Medicine, vol. 8, no. 4, pp. 173-176, 1999.

[7] L. J. Bodner, J. L. Nosher, C. Gribbin, R. L. Siegel, S. Beale, and W. Scorza, "Balloon-assisted occlusion of the internal iliac arteries in patients with placenta accreta/percreta," CardioVascular and Interventional Radiology, vol. 29, no. 3, pp. 354-361, 2006.

[8] K. W. Yi, M.-J. Oh, T.-S. Seo, K. A. So, Y. C. Paek, and H.-J. Kim, "Prophylactic hypogastric artery ballooning in a patient with complete placenta previa and increta," Journal of Korean Medical Science, vol. 25, no. 4, pp. 651-655, 2010.

[9] NCRP, "Risk estimates for radiation protection," NCRP Report 115, National Council on Radiation Protection and Mesurements, Bethesda, Md, USA, 1993.

[10] International Commission on Radiological Protection, "Pregnancy and medical radiation," Annals of the ICRP, vol. 30, no. 3-4, pp. 1-43, 2000.

[11] J. V. Neel, W. J. Schull, A. A. Awa et al., "The children of parents exposed to atomic bombs: estimates of the genetic doubling 
dose of radiation for humans," The American Journal of Human Genetics, vol. 46, no. 6, pp. 1053-1072, 1990.

[12] L. T. Uradomo, E. M. Goldberg, and P. E. Darwin, "Timelimited fluoroscopy to reduce radiation exposure during ERCP: a prospective randomized trial," Gastrointestinal Endoscopy, vol. 66, no. 1, pp. 84-89, 2007.

[13] J. Valentin, "Radiation and your patient: a guide for medical practitioners," Annals of the ICRP, vol. 31, no. 4, pp. 1-52, 2001.

[14] M. A. Belfort, "Indicated preterm birth for placenta accreta," Seminars in Perinatology, vol. 35, no. 5, pp. 252-256, 2011. 


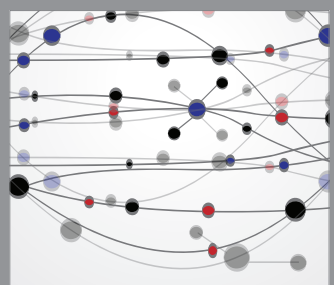

The Scientific World Journal
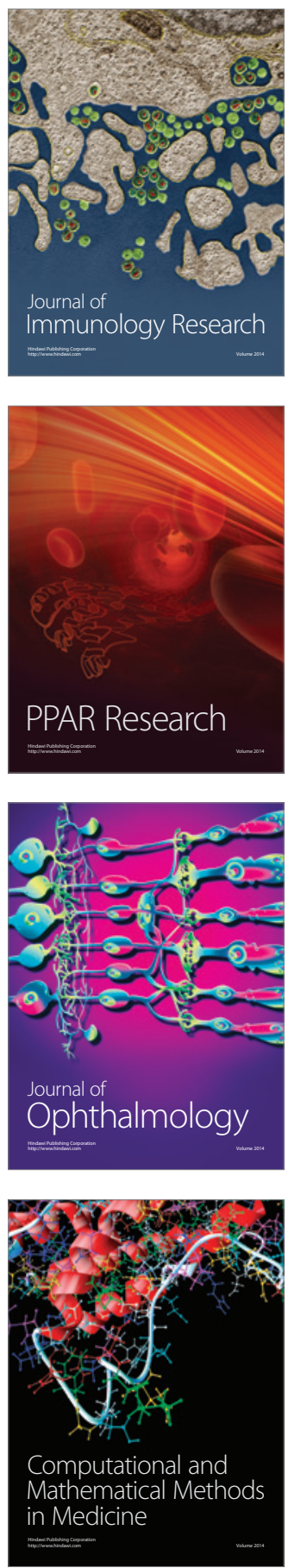

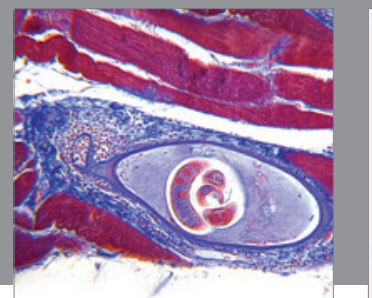

Gastroenterology

Research and Practice
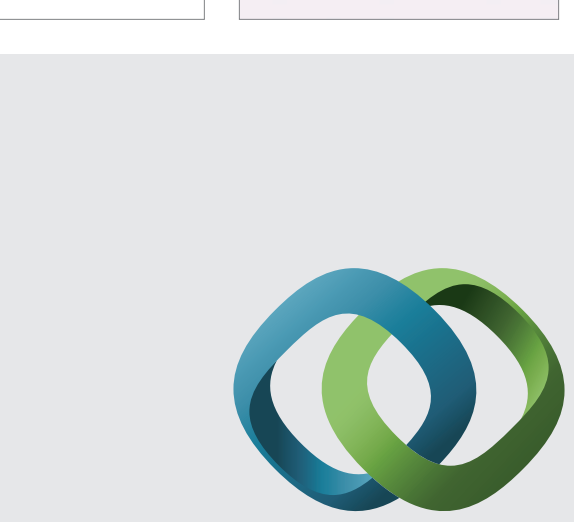

\section{Hindawi}

Submit your manuscripts at

http://www.hindawi.com
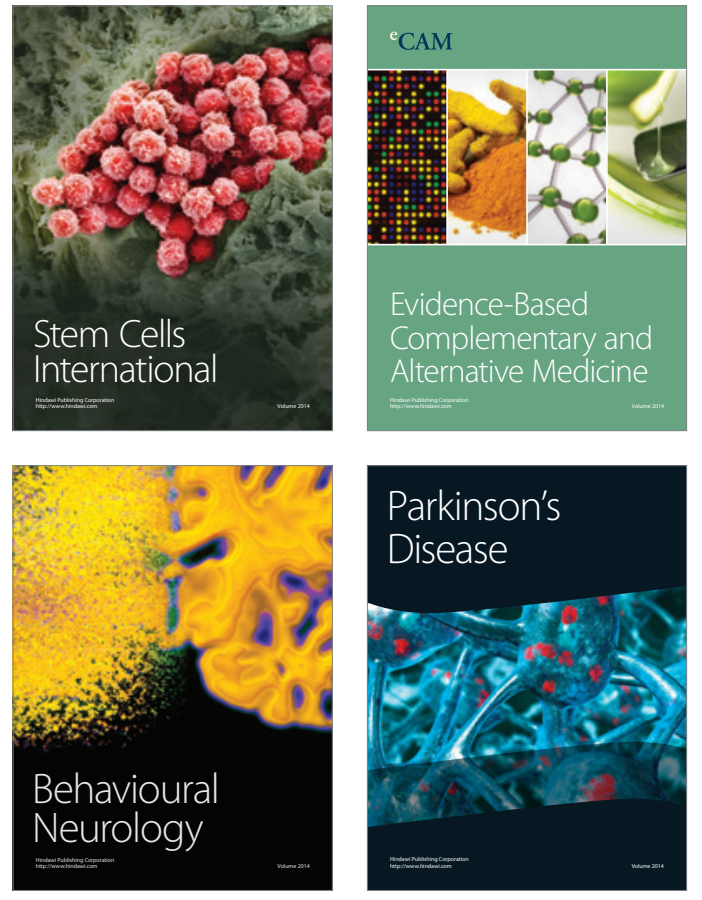
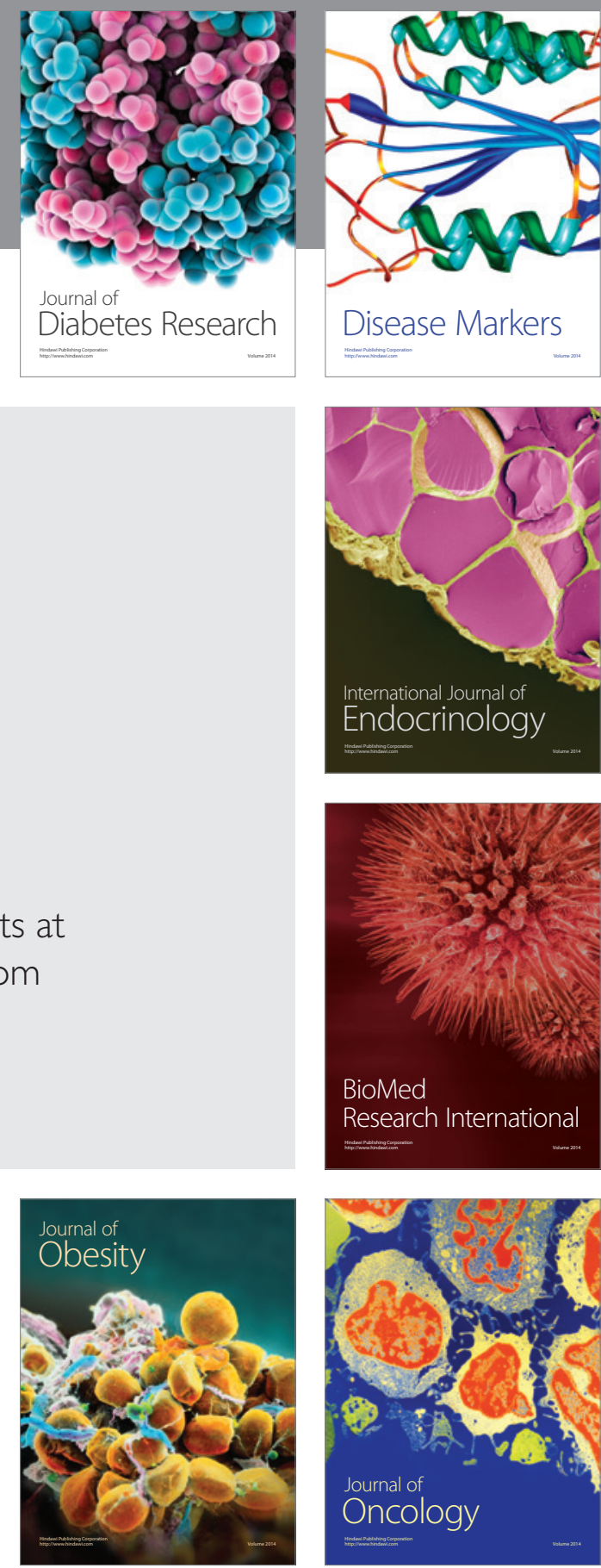

Disease Markers
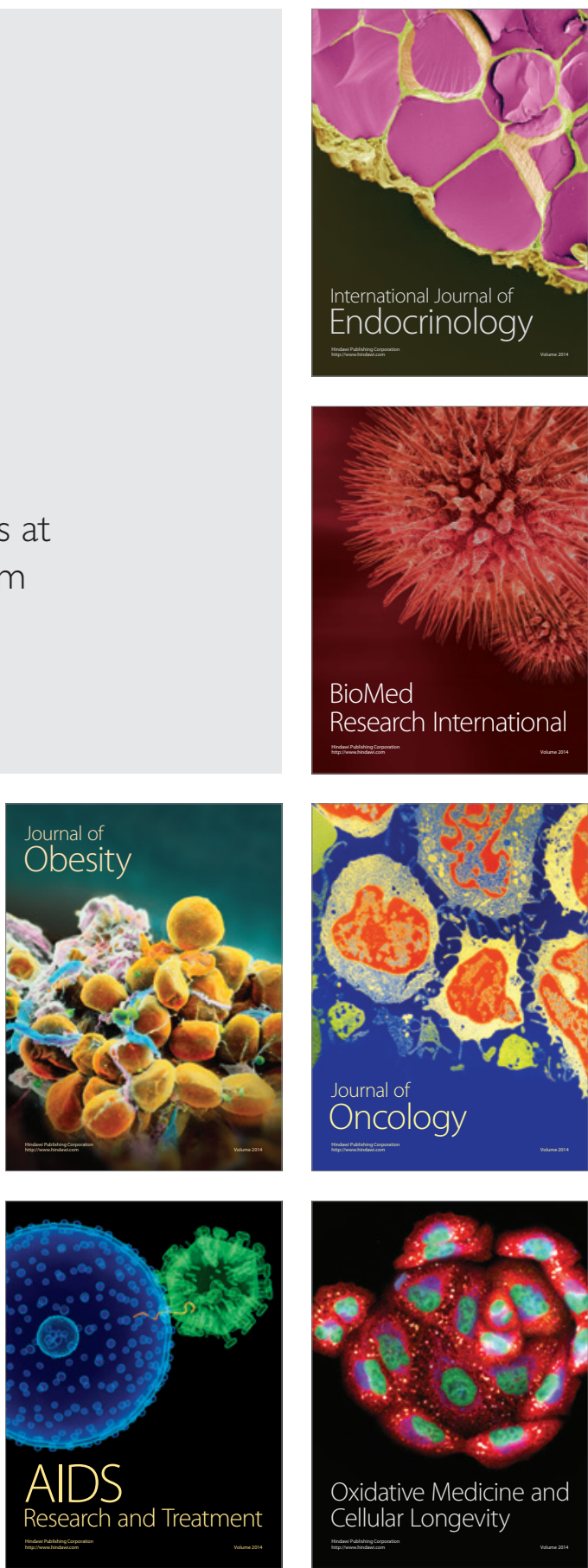